INFECTION CONTROL AND HOSPITAL EPIDEMIOLOGY OCTOBER 2014, vol. 35, No. S3

\title{
Can Additional Information Be Obtained from Claims Data to Support Surgical Site Infection Diagnosis Codes?
}

\author{
David K. Warren, MD, MPH; ${ }^{1}$ Katelin B. Nickel, MPH;' Anna E. Wallace, MPH; ${ }^{2}$ Daniel Mines, MD, MSCE; \\ Victoria J. Fraser, MD; ${ }^{1}$ Margaret A. Olsen, $\mathrm{PhD}, \mathrm{MPH}^{1,3}$
}

\begin{abstract}
овјестіve. International Classification of Diseases, Ninth Revision, Clinical Modification (ICD-9-CM) diagnosis codes are increasingly used to identify healthcare-associated infections, often with insufficient evidence demonstrating validity of the codes used. Absent medical record verification, we sought to confirm a claims algorithm to identify surgical site infections (SSIs) by examining the presence of clinically expected SSI treatment.
\end{abstract}

METHODS. We performed a retrospective cohort study, using private insurer claims data from persons less than 65 years old with ICD9-CM procedure or Current Procedure Terminology (CPT-4) codes for anterior cruciate ligament (ACL) reconstruction from January 2004 through December 2010. SSIs occurring within 90 days after ACL reconstruction were identified by ICD-9-CM diagnosis codes. Antibiotic utilization, surgical treatment, and microbiology culture claims within 14 days of SSI codes were used as evidence to support the SSI diagnosis.

RESULTS. Of 40,702 procedures, $401(1.0 \%)$ were complicated by SSI, $172(0.4 \%)$ of which were specifically identified as septic arthritis. Most SSIs were associated with an inpatient admission (232/401 [58\%]), and/or surgical procedure(s) for treatment (250/401 [62\%]). Temporally associated antibiotics, surgical treatment procedures, and cultures were present for $84 \%$ (338/401), 61\% (246/401), and 59\% (238/401), respectively. Only 5.7\% (23/401) of procedures coded for SSI after the procedure had no antibiotics, surgical treatments, or cultures within 14 days of the SSI claims.

CONCLUSIONS. More than $94 \%$ of patients identified by our claims algorithm as having an SSI received clinically expected treatment for infection, including antibiotics, surgical treatment, and culture, suggesting that this algorithm has very good positive predictive value. This method may facilitate retrospective SSI surveillance and comparison of SSI rates across facilities and providers.

Infect Control Hosp Epidemiol 2014;35(S3):S124-S132

Increasingly, billing or claims data are being used to identify healthcare-associated infections, including surgical site infections (SSIs). The accuracy of International Classification of Diseases, Ninth Revision, Clinical Modification (ICD-9-CM) diagnosis codes to identify infections has been reported in a number of studies with varying results, depending on the surgical procedures studied and the diagnosis codes used to indicate infection. ${ }^{1-10}$ Anterior cruciate ligament (ACL) reconstruction is a surgical procedure for which there has been no validation of the ICD-9-CM diagnosis codes to identify subsequent SSIs.

Infection following ACL reconstruction is rare, and most reports in the literature are single-center studies with a small number of infections. Among studies with 5 or more cases of septic arthritis or SSI, reported infection rates range from $0.14 \%$ to $1.96 \% ;^{11-25} 12$ of the 15 studies reported rates of less than $1.0 \%$. Infection after ACL reconstruction is uncommon, but it can lead to poor outcomes, including articular cartilage destruction, arthrofibrosis, loss of range of motion, and reduced activity level. ${ }^{11-15,17,23,26}$ While there are no defined treatment guidelines, typical treatment of $\mathrm{ACL}$ reconstruction-related infection includes antibiotics and arthroscopic or open drainage of the knee. ${ }^{27.28}$

We sought to determine whether additional information could be obtained from administrative claims data to support the diagnosis of SSI following ACL reconstruction. In the absence of a gold standard such as medical chart review, we used clinically expected treatments available in the administrative claims-specifically, antibiotic utilization, use of microbiology cultures, and surgical treatment for infection-to support the coding of SSI.

\section{METHODS}

\section{Data Source}

We conducted a retrospective cohort study, using the HealthCore Integrated Research Database (HIRD). Individuals represented in the HIRD include lives from 14 WellPoint-

Affiliations: 1. Division of Infectious Diseases, Department of Medicine, Washington University, St. Louis, Missouri; 2. HealthCore, Wilmington, Delaware; 3. Division of Public Health Sciences, Department of Surgery, Washington University, St. Louis, Missouri.

Received May 19, 2014; accepted May 25, 2014; electronically published September 15, 2014.

(C) 2014 by The Society for Healthcare Epidemiology of America. All rights reserved. 0899-823X/2014/35S3-0018\$15.00. DOI: 10.1086/677830 
affiliated plans. WellPoint is an independent licensee of the Blue Cross and Blue Shield (BCBS) Association and serves its members as the Blue Cross licensee for California and the BCBS licensee for Colorado, Connecticut, Georgia, Indiana, Kentucky, Maine, Missouri (excluding 30 counties in the Kansas City area), Nevada, New Hampshire, New York (as the BCBS licensee in 10 New York City metropolitan and surrounding counties and as the Blue Cross or BCBS licensee in selected upstate counties only), Ohio, Virginia (excluding the northern Virginia suburbs of Washington, DC), and Wisconsin. Thirteen plans were used for this research. Data in the HIRD include all fully adjudicated claims submitted for reimbursement from providers, facilities, and outpatient pharmacies and are linked to health plan enrollment information.

Fully insured members from 6 months to 64 years of age who were enrolled in a health plan that included medical coverage of hospital and physician services were eligible for selection into the study cohort. Prescription drug coverage was also required in order to assess antibiotic utilization. Exclusions included members with an ICD-9-CM diagnosis code or prescription claim indicating HIV-positive status at any time (for patient privacy) and members likely to have incomplete data (eg, those enrolled in a capitated plan or in multiple plans at the time of surgery). We also excluded members enrolled in a plan with hospital coverage only, since up to $60 \%$ of SSIs are identified and managed in the ambulatory setting. ${ }^{29}$ Medical and pharmacy claims were restricted to paid claims.

The claims data available for this study contained up to 5 ICD-9-CM diagnosis codes per claim. Facility (hospital or ambulatory) claims included Uniform Billing (UB-92/UB-04) revenue and Healthcare Common Procedure Coding System (HCPCS) codes. Hospitals included up to 5 ICD-9-CM procedure codes per claim, while ambulatory facilities reported Current Procedure Terminology (CPT-4) procedure codes. Provider claims included both CPT-4 and HCPCS codes.

We utilized the American Hospital Association Annual Survey of Hospitals (Health Forum) and the Outpatient Surgery Center Profiling Solution data (IMS Health) in order to determine whether the ACL reconstruction was performed at a hospital or a freestanding ambulatory surgery center. The facility information from these two data sources was matched to the operative facility via National Provider Identifier codes, where available; otherwise, matching was performed with facility name and address fields.

\section{ACL Reconstruction Patient Population}

We identified ACL reconstruction procedures performed on an inpatient or outpatient basis at a hospital or a freestanding ambulatory surgery center by using ICD-9-CM and CPT-4 procedure codes from all facility (other than home health agencies) and provider claims among members eligible for cohort entry aged 6 months to 64 years between January 1, 2004, and December 31, 2010 (Table 1). The ACL recon- struction patient population was refined by excluding procedures likely to have erroneous claims for ACL reconstruction, procedures in members whose enrollment ended on the day of the surgical procedure, complicated procedures or procedures in patients considered medically complicated, and procedures in which the surgery date could not be determined from the available information in the claims (see below for description).

\section{Identification and Exclusion of Erroneous Claims for ACL Reconstruction}

We created an algorithm to identify problematic claims, which we defined as facility claims that contained apparent CPT-4, HCPCS, or UB-04 revenue codes truncated to 4 digits and populated in the fields reserved for ICD-9-CM procedure codes. This error appeared to occur during processing of certain types of non-inpatient facility claims (A. E. Wallace and $\mathrm{M}$. A. Olsen, unpublished data). Claims in which an $\mathrm{ACL}$ procedure code was the only procedure code present, with no other claims submitted for the same date, were also classified as problematic and excluded.

\section{Exclusion of Complicated Patients and Procedures}

The overall aim of this research study was to estimate the risk of SSI after ACL procedures by surgical facility type. For this reason, we excluded ACL reconstruction procedures performed in medically complicated patients who would be very unlikely to undergo surgery in an ambulatory setting and would have a risk profile very different from that of most ACL patients. We defined medically complicated patients as persons with endstage renal disease or septicemia between 7 days before and 1 day after the ACL procedure date (Table 1).

We also excluded ACL reconstruction procedures performed at the time of or after another surgical procedure during the same admission, since these procedures would be complex and attribution of an SSI to a particular procedure would not be possible. These additional surgical procedures were identified via CPT-4 and ICD-9-CM procedure codes from the National Healthcare Safety Network (NHSN) list of procedures for SSI surveillance. ${ }^{30}$ We also excluded ACL reconstruction procedures in which any of the following procedures were coded within 7 days of ACL surgery, since these represent more complex ACL reconstructions: partial ostectomy, limb-lengthening procedure, internal fixation of bone of leg, open reduction of fracture of leg, or patellectomy (Table 1).

Finally, we excluded ACL reconstructions performed on or after calendar day 3 (where day 1 was the day of admission) of an inpatient admission. The rationale for choosing the day 3 cutoff is that scheduled, elective surgical procedures are typically performed either on the day of admission or on the following day. A surgical procedure performed on hospital day 3 or later would be unlikely to be the primary reason for admission. Therefore, these patients would not have had 
TABLE 1. Codes Used to Identify Anterior Cruciate Ligament (ACL) Reconstruction, Procedure Exclusions, and Evidence for Surgery

\begin{tabular}{|c|c|c|c|c|}
\hline & CPT-4 codes & $I C D-9-C M$ procedure codes & UB-04 revenue codes & $I C D-9-C M$ diagnosis codes \\
\hline \multicolumn{5}{|l|}{$\begin{array}{l}\text { Codes used to identify ACL reconstruc- } \\
\text { tion procedure }\end{array}$} \\
\hline ACL reconstruction & $\begin{array}{l}27407,27409,27427-27429 \\
29888\end{array}$ & $81.43,81.45$ & & \\
\hline \multicolumn{5}{|l|}{$\begin{array}{l}\text { Codes used for ACL reconstruction } \\
\text { exclusion }\end{array}$} \\
\hline End-stage renal disease & & & & $\begin{array}{r}585.6, \mathrm{~V} 45.1, \mathrm{~V} 45.11, \mathrm{~V} 45.12 \\
\mathrm{~V} 56.0, \mathrm{~V} 56.1, \mathrm{~V} 56.2, \mathrm{~V} 56.8\end{array}$ \\
\hline Septicemia & & & & $038.0-038.9,790.7$ \\
\hline $\begin{array}{l}\text { Partial ostectomy, limb lengthening } \\
\text { procedure, internal fixation of bone } \\
\text { of leg, open reduction of fracture of } \\
\text { leg, patellectomy }\end{array}$ & $\begin{array}{l}27228,27236,27244,27245, \\
27248,27254,27269,27350, \\
27506,27507,27511,27513 \\
27514,27535,27536,27540 \\
27758,27759,27766,27769 \\
27784,27792,27814,27822 \\
27823,27826-27828\end{array}$ & $\begin{array}{l}77.85,77.86,77.87,77.89 \\
78.35,78.37,78.39,78.55 \\
78.56,78.57,78.59,79.25 \\
79.26,79.35,79.36,79.55 \\
79.56\end{array}$ & & \\
\hline \multicolumn{5}{|l|}{$\begin{array}{l}\text { Codes used as additional evidence for } \\
\text { ACL reconstruction surgery }\end{array}$} \\
\hline Anesthesia & $01320,01380,01400$ & & & \\
\hline Tendon graft & 20924,20926 & & & \\
\hline Surgery-related revenue codes & & & $\begin{array}{l}0201,0360,0361,0369 \\
0370,0379,0490,0499 \\
0963,0964,0975\end{array}$ & \\
\hline
\end{tabular}

Note. CPT-4, Current Procedural Terminology, 4th edition; ICD-9-CM, International Classification of Diseases, Ninth Revision, Clinical Modification; UB, Uniform Billing. 
TABLE 2. Codes Used to Identify Surgical Site Infection (SSI) following Anterior Cruciate Ligament Reconstruction

\begin{tabular}{ll}
\hline Knee-specific infection codes to identify SSI & \multicolumn{1}{c}{ ICD-9-CM diagnosis codes } \\
Septic arthritis & $711.06,711.96$ \\
Other infection to lower leg or joint prosthesis & $711.66,730.06,730.16,730.26,730.96,996.66,996.67$ \\
General infection codes to identify SSI & $998.5-998.59$ \\
Postoperative infection & 728.0 \\
Infective myositis & \\
\hline NOTE. ICD-9-CM, International Classification of Diseases, Ninth Revision, Clinical Modification.
\end{tabular}

the opportunity to have the surgery performed at a freestanding ambulatory surgery center.

\section{Establishing the Surgery Date and Use of Supporting Evidence for Surgery}

ACL reconstruction dates within 7 days were collapsed into a single surgery due to potential inaccuracy in dates, particularly on provider claims. ${ }^{31}$ In these instances, we compared facility and provider surgery dates and incorporated supplemental evidence (eg, claims for anesthesia and tendon graft procedures) from unique providers to determine the most likely surgery date. We excluded ACL reconstruction procedures coded by either a provider or a facility only, unless there was additional evidence that a surgical procedure took place, that is, claims for anesthesia services, tendon graft procedure, or a surgery-related UB-04 revenue code (Table 1).

\section{Identification of SSIs}

Claims for SSIs first recorded from 2 to 90 days after eligible procedures were identified via ICD-9-CM diagnosis codes (Table 2). We excluded individual SSI claims with locations that were not consistent with a provider diagnosis (eg, lab- oratory, patient's home) and those with CPT-4 codes for pathology services (88104-88399). This was done to avoid capturing an SSI that may have been a rule-out or a working diagnosis.

\section{Timing of SSI}

The date of SSI onset was defined according to the timing and location of diagnosis. For SSIs coded by an inpatient facility during the original operative admission, we assigned the date of SSI to the discharge date if the difference between the discharge and admission dates was greater than or equal to 2 days. For SSIs diagnosed during a subsequent inpatient admission, the date of SSI onset was assumed to be the date of hospital admission. For SSIs diagnosed by a provider or in an ambulatory setting, the onset date was defined as the first service date with an ICD-9-CM diagnosis code for SSI.

The observation period for development of SSIs was through 90 days after surgery, with earlier censoring for the end of insurance enrollment, subsequent $\mathrm{ACL}$ reconstruction, knee replacement, or other knee or leg surgery (ie, partial ostectomy, limb-lengthening procedure, internal fixation of bone of leg, open reduction of fracture of leg, or patellec-

TAвLE 3. Codes Used as Supplemental Evidence for Surgical Site Infection (SSI) following Anterior Cruciate Ligament Reconstruction

ICD-9-CM or CPT-4

procedure codes

Antibiotic

Knee-specific surgical treatment for SSI

$27301,27303,27310,27330$,

27331, 27334, 27335,

27360, 29870, 29871,

$29873,29875,29876$,

$29884,80.06,80.16,80.26$

General surgical treatment for SSI

Microbiology culture
10060, 10061, 10180, 20000, 20005,20680

$87040,87070,87071,87073$, $87075,87076,87077$

Note. ICD-9-CM, International Classification of Diseases, Ninth Revision, Clinical Modification; CPT-4, Current Procedural Terminology, 4 th edition. 
tomy). In patients with subsequent surgeries, we censored 1 day after the subsequent surgery. Non-knee-specific ICD-9$C M$ diagnosis codes for infection ( $\mathrm{eg}, 998.59)$ were not classified as SSIs if they were first coded after a subsequent nonknee NHSN surgery within 90 days.

An ICD-9-CM diagnosis code for an SSI from 30 days before to 1 day after surgery was considered to indicate a preexisting infection. These ACL procedures were excluded from the study, since our goal was to identify incident cases of SSI.

\section{Evidence Supporting the Diagnosis of SSI}

Prescription and medical claims for antibiotics, ICD-9-CM and CPT-4 procedure codes for surgical treatment, and CPT4 codes for microbiology cultures were used to support the occurrence of an SSI (Table 3). Among persons with an incident SSI ICD-9-CM diagnosis code attributable to the ACL procedure, we considered antibiotic, surgical treatment, and culture claims 1-90 days after ACL reconstruction that were within 14 days of a date of an SSI diagnosis code and before applicable censoring to be supporting evidence for the coded SSI.

\section{Statistical Analysis}

All statistical analyses used the $\chi^{2}$ test. All data management and analyses used SAS, version 9.3 (SAS Institute). This study was approved by the Washington University Human Research Protection Office.

\section{RESULTS}

A total of $41,837 \mathrm{ACL}$ reconstruction procedures met all eligibility criteria and were identified during the 7-year study period. The number of distinct procedures was reduced to 40,702 procedures among 38,883 patients after removal of procedures with no supporting evidence for the procedure ( $n=686)$, complicated ACL procedures and procedures in medically complicated patients $(n=393)$, and ACL reconstructions performed at the time of a preexisting SSI ( $n=$ 56). More than one ACL reconstruction was performed during the study period in $4.4 \%$ of patients. The procedures were evenly distributed over the study years. Most procedures were performed as day surgery at a hospital or at a freestanding ambulatory surgery center. Most ACL reconstruction procedures involved males, and the median age was 29 years (range, 2-64 years; Table 4).

SSIs were identified by ICD-9-CM diagnosis codes after $401(1.0 \%$ [95\% confidence interval (CI), $0.9 \%-1.1 \%])$ procedures. The median time to onset was 20 days (interquartile range, 10-33 days), with 293 (73\%) SSIs identified up to 30 days after the ACL reconstruction procedure. Fifty-four percent $(n=218)$ had at least one knee-specific SSI code, as defined in Table 2. Fifty-eight percent $(n=232)$ of patients had a hospital admission associated with their SSIs. A total of 250 patients $(62 \%)$ had one or more surgical procedures
TABle 4. Characteristics of Anterior Cruciate Ligament Reconstruction Procedures in 38,883 Patients

\begin{tabular}{lc}
\hline Characteristic & $n(\%)$ \\
\hline Total procedures & 40,702 \\
Age, median (range), years & $29(2-64)$ \\
Age <18 years & $7,436(18.3)$ \\
Male & $24,490(60.2)$ \\
Location of procedure & \\
$\quad$ Inpatient & $1,953(4.8)$ \\
Day surgery at hospital & $15,769(38.7)$ \\
Ambulatory surgery center & $12,526(30.8)$ \\
Missing facility type & $10,454(25.7)$ \\
Procedures by year & \\
2004 & $5,664(13.9)$ \\
2005 & $5,874(14.4)$ \\
2006 & $6,041(14.8)$ \\
2007 & $5,989(14.7)$ \\
2008 & $6,134(15.1)$ \\
2009 & $5,751(14.1)$ \\
2010 & $5,249(12.9)$ \\
\hline
\end{tabular}

NOTE. Unless otherwise specified, data are no. (\%) of procedures.

a "Inpatient" and "day surgery" represent matches to a facility in the American Hospital Association (AHA) Annual Survey of Hospitals (Health Forum); inpatient classification was based on an inpatient designation in the HealthCore claims data. "Ambulatory surgery center" represents a match to a facility in the IMS Health Outpatient Surgery Center Profiling Solution data.

b Facility type was classified as missing because there was no facility claim for procedure $(n=4,065)$, no match to a facility in the AHA Annual Survey of Hospitals or the IMS Health Outpatient Surgery Center Profiling Solution data $(n=6,366)$, or a match to multiple facilities $(n=23)$.

for treatment (median, 1; range, $0-5$ ). Among those with an SSI, $43 \%(n=172)$ had at least one code for septic arthritis, for an overall incidence of septic arthritis of $0.4 \%$ (95\% CI, $0.4 \%-0.5 \%)$. Persons with septic arthritis were more likely to have had an inpatient admission at the time of infection and more likely to have had surgical treatment than persons coded for SSI but not septic arthritis (Table 5).

Overall, $84.3 \%(338 / 401)$ of SSIs had a temporally associated antibiotic claim, 61.4\% (246/401) had a temporally associated claim coded for surgical treatment, and 59.4\% (238/401) had a temporally associated claim for microbiology culture. In total, 155/401 (38.7\%) SSIs had 3 types of supporting evidence (ie, antibiotics, surgical treatment, and culture), as seen in the center of the overlapping circles in Figure $1,134(33.4 \%)$ had 2 types of evidence (ie, 70 SSIs had surgical treatment and antibiotics, 56 SSIs had antibiotics and culture, and 8 SSIs had surgical treatment and culture), and 89 $(22.2 \%)$ had one type of supporting evidence. Only $23(5.7 \%)$ SSIs had no additional claim for an antibiotic, surgical treatment, or culture to support the diagnosis of SSI, for a positive predictive value (PPV) of $94.3 \%$ (378/401; Figure 1). The 
TA B LE 5. Characteristics of Surgical Site Infection (SSI) and Septic Arthritis following 40,702 Anterior Cruciate Ligament Reconstruction Procedures

\begin{tabular}{|c|c|c|c|c|}
\hline Characteristic & Total SSI & Septic arthritis & SSI, no septic arthritis & $P^{\mathrm{a}}$ \\
\hline Total & 401 & 172 & 229 & \\
\hline Incidence, $\%$ & 1.0 & 0.4 & 0.6 & \\
\hline Infection coded during an inpatient admission, $n(\%)$ & $232(57.9)$ & $137(79.7)$ & $95(41.5)$ & $<.01$ \\
\hline Surgical treatment for SSI in postoperative period, $n(\%)$ & $250(62.3)$ & $149(86.6)$ & $101(44.1)$ & $<.01$ \\
\hline
\end{tabular}

a As determined by the $\chi^{2}$ test.

"See "Methods" for specific ICD-9-CM (International Classification of Diseases, Ninth Revision, Clinical Modification) and CPT-4 (Current Procedural Terminology, 4th ed.) procedure codes used for surgical treatment.

PPV of our SSI algorithm was $89.5 \%$ (359/401) when only antibiotics and surgical treatment were considered as supporting evidence. The proportion of antibiotic, surgical treatment, and culture claims data associated with SSI did not vary significantly on the basis of whether the onset of SSI was up to 30 days or 31-90 days after the ACL reconstruction procedure (Table 6).

Among the 338 persons with an SSI and a temporally associated antibiotic, the most common classes of antibiotics prescribed were cephalosporins (59\%), vancomycin (28\%), and fluoroquinolones $(21 \%)$. Among the 246 persons with an SSI and an associated surgical procedure for treatment, $164(67 \%)$ had an arthroscopy procedure, $101(41 \%)$ had an arthrotomy procedure or removal of implant, and 92 (37\%) had another incision-and-drainage procedure.

\section{DISCUSSION}

The use of administrative data to identify healthcare-associated infection is challenging, but these data can be an important resource for relatively rare events such as SSIs. Some authors have concluded that billing and claims data cannot be reliably used for SSI surveillance. ${ }^{4,9,10}$ We found that $94.3 \%$ of patients identified as having an SSI by our rigorous claims algorithm also received clinically expected treatment for infection; a more conservative PPV estimate excluding culture was still very high, at $89.5 \%$. While we could not confirm the SSIs with medical chart review, our results suggest that the claims algorithm we used to identify SSIs has very good PPV.

We used only ICD-9-CM diagnosis codes that were specific to SSIs and/or were consistent with the NHSN clinical SSI

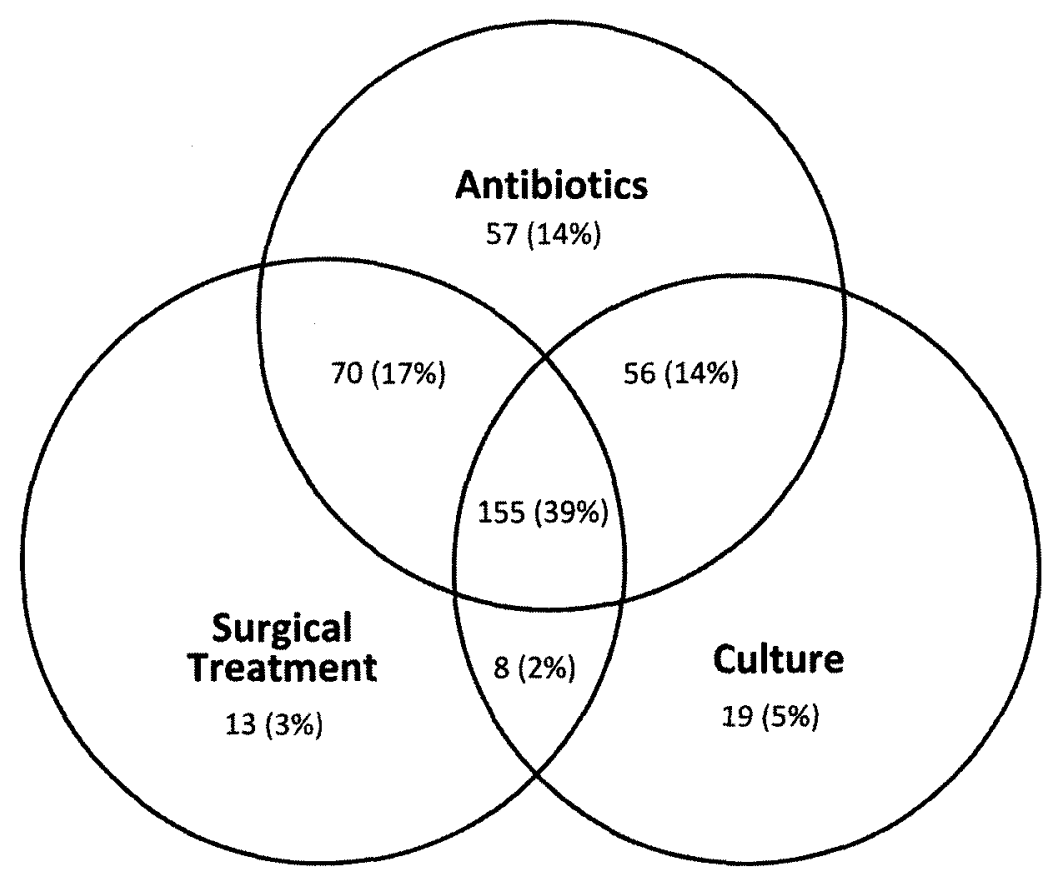

FIGURE 1. Description of supporting evidence for 401 cases of surgical site infection (SSI) after anterior cruciate ligament reconstruction procedures. Twenty-three (5.7\%) of the 401 SSIs identified by ICD-9-CM (International Classification of Diseases, Ninth Revision, Clinical Modification) diagnosis codes had no subsequent claims for antibiotics, surgical treatment for SSI, or microbiology cultures. 


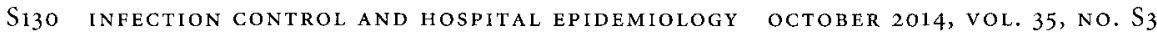

TABLE 6. Surgical Treatment and Use of Antibiotics and Culture within 14 Days of Anterior Cruciate Ligament ReconstructionAssociated Surgical Site Infection (SSI) by SSI Onset

\begin{tabular}{|c|c|c|c|c|}
\hline Characteristic & Total SSI, $n(\%)$ & $\begin{array}{c}\text { SSI onset } \leq 30 \text { days from } \\
\text { procedure, } n(\%)\end{array}$ & $\begin{array}{c}\text { SSI onset }>30 \text { days from } \\
\text { procedure, } n(\%)\end{array}$ & $P^{\mathrm{a}}$ \\
\hline Total & 401 & 293 & 108 & \\
\hline \multicolumn{5}{|l|}{ At least 1 claim for } \\
\hline Antibiotics & $338(84.3)$ & $250(85.3)$ & $88(81.5)$ & .35 \\
\hline Surgical treatment & $246(61.4)$ & $178(60.8)$ & $68(63.0)$ & .69 \\
\hline Culture & $238(59.4)$ & $173(59.0)$ & $65(60.2)$ & .84 \\
\hline $\begin{array}{l}\text { Antibiotics, surgical treatment, } \\
\text { and/or culture }\end{array}$ & $378(94.3)$ & $278(94.9)$ & $100(92.6)$ & .38 \\
\hline
\end{tabular}

"As determined by the $\chi^{2}$ test.

definition. Studies that used specific SSI ICD-9-CM diagnosis codes (eg, 998.5, 998.51, and 998.59) (1-3 $^{1-3}$ were more likely to report a higher PPV than studies using a larger range of diagnosis codes including ones less specific for SSI. ${ }^{4,9,10}$ We also used available information to distinguish preexisting and incident infections and censored at the time of any subsequent procedures. This censoring reduced the likelihood of attributing an SSI after a subsequent surgery to the index ACL reconstruction, which has been reported previously as a source of misclassification bias when using administrative data. ${ }^{3,10}$

In our large, geographically diverse study population, we found the incidence of SSI following ACL reconstruction to be $1.0 \%$. This rate is higher than that in 12 of the 15 studies we identified in the published English-language literature. ${ }^{11,13-18,20,21,23-25}$ There are several potential reasons for our findings. We used claims data from across the spectrum of care, rather than from readmission ${ }^{20}$ or single-center medical record review, to identify infections. ${ }^{11-19,22-25}$ The inclusion of outpatient claims has been shown by others to capture at least twice as many SSIs as inpatient surveillance alone. ${ }^{7,29}$ Only 2 studies that reported low SSI rates were from multiple institutions. Maletis et al. ${ }^{21}$ reported an overall SSI rate of $0.46 \%(0.3 \%$ deep SSI, $0.1 \%$ superficial SSI), using a Kaiser Permanente registry, with all outcomes verified by chart review. Jameson et al. ${ }^{20}$ utilized data from the English National Health Service and reported a rate of $0.25 \%$ for deep infection within 30 days and $0.75 \%$ for wound complication (infection and hematoma), but identification of complications relied solely on hospital readmissions. Another explanation for the lower SSI rates in the literature is that most studies included only more severe SSIs (eg, septic arthritis). ${ }^{11-20,22,24,25}$ For example, in 12 published studies, all patients with reported SSIs received intravenous antibiotics, ${ }^{11-19,22,24,25}$ while in another 12 studies, all cases with SSIs required surgical treatment. ${ }^{11-20,22,25}$ While the incidence of total SSIs in our current study is about twice that of reported rates, our reported incidence rates of septic arthritis (0.4\%) and more severe infections (ie, infections requiring hospital admission [0.6\%] and/or surgical treatment $[0.6 \%]$ ) are consistent with the SSI rates reported in the literature.

Limitations of claims data for SSI surveillance include is- sues common to secondary analysis of data collected for other purposes (ie, billing and reimbursement). Therefore, some data elements that are important for SSI risk prediction surveillance, such as procedure dates, may be less accurate, since they do not affect reimbursement. There is also likely undercoding of SSIs, particularly minor infections, during the 90-day global surgical provider reimbursement period..$^{32}$ Thus, our calculation of SSI incidence after ACL reconstruction likely underestimates the true infection rate, since minor infections that occurred within the global reimbursement period may not have been coded. In addition, our findings may not be generalizable to all ACL reconstruction procedures, because we limited our surgical population to less complex procedures. While medical chart review is considered the gold standard for validation, medical records were not available for private insurer claims data study. However, medical chart review is often limited to single-center studies, while our data represent hundreds of facilities and providers, which increases the generalizability of our findings. Future studies could use medical chart review as the gold standard to confirm our findings but would require procedures from various practice settings (eg, urban/rural, ambulatory/inpatient). Our use of temporally associated clinical treatment for SSI to support coding of SSI is reproducible and allows patients to be tracked across the spectrum of care.

More than $94 \%$ percent of patients identified by our claims algorithm as having an SSI received clinically expected treatment for infection, suggesting that the algorithm has very good PPV. This method may facilitate retrospective surveillance and comparison of SSI rates across facilities and providers.

\section{ACKNOWLEDGMENTS}

We thank Cherie Hill for database and computer management support.

Financial support. Funding for this project was provided by the Agency for Healthcare Research and Quality, grant 5R01HS019713 (to M.A.O.).

Potential conflicts of interest. M.A.O. reports consultant work with Pfizer and Sanofi Pasteur and grant funding through Cubist Pharmaceuticals and Sanofi Pasteur for work outside the submitted manuscript. All other authors report no conflicts of interest relevant to this article. All authors submitted 
the ICMJE Form for Disclosure of Potential Conflicts of Interest, and the conflicts that the editors consider relevant to this article are disclosed here.

Address correspondence to: Margaret A. Olsen, PhD, MPH, Division of Infectious Diseases, Campus Box 8051, Washington University, 660 South Euclid Avenue, St. Louis, MO 63110 (molsen@dom.wustl.edu).

The findings and conclusions in this document are those of the authors, who are responsible for its content, and do not necessarily represent the views of the Agency for Healthcare Research and Quality (AHRQ). No statement in this report should be construed as an official position of AHRQ or of the U.S. Department of Health and Human Services.

\section{REFERENCES}

1. Yokoe DS, Noskin GA, Cunningham SM, et al. Enhanced identification of postoperative infections among inpatients. Emerg Infect Dis 2004;10(11):1924-1930.

2. Bolon MK, Hooper D, Stevenson KB, et al. Improved surveillance for surgical site infections after orthopedic implantation procedures: extending applications for automated data. Clin Infect Dis 2009;48(9):1223-1229.

3. Olsen MA, Fraser VJ. Use of diagnosis codes and/or wound culture results for surveillance of surgical site infection after mastectomy and breast reconstruction. Infect Control Hosp Epidemiol 2010;31(5):544-547.

4. West J, Khan Y, Murray DM, Stevenson KB. Assessing specific secondary ICD-9-CM codes as potential predictors of surgical site infections. Am I Infect Control 2010;38(9):701-705.

5. Calderwood MS, Ma A, Khan YM, et al. Use of Medicare diagnosis and procedure codes to improve detection of surgical site infections following hip arthroplasty, knee arthroplasty, and vascular surgery. Infect Control Hosp Epidemiol 2012;33(1):4049.

6. Hollenbeak CS, Boltz MM, Nikkel LE, Schaefer E, Ortenzi G, Dillon PW. Electronic measures of surgical site infection: implications for estimating risks and costs. Infect Control Hosp Epidemiol 2011;32(8):784-790.

7. Lawson EH, Louie R, Zingmond DS, et al. A comparison of clinical registry versus administrative claims data for reporting of 30-day surgical complications. Ann Surg 2012;256(6):973981.

8. Yokoe DS, Khan Y, Olsen MA, et al. Enhanced surgical site infection surveillance following hysterectomy, vascular, and colorectal surgery. Infect Control Hosp Epidemiol 2012;33(8):768773.

9. Julian KG, Brumbach AM, Chicora MK, et al. First year of mandatory reporting of healthcare-associated infections, Pennsylvania: an infection control-chart abstractor collaboration. Infect Control Hosp Epidemiol 2006;27(9):926-930.

10. Stevenson KB, Khan Y, Dickman J, et al. Administrative coding data, compared with CDC/NHSN criteria, are poor indicators of health care-associated infections. Am J Infect Control 2008; $36(3): 155-164$.

11. Van Tongel A,, Stuyck J, Bellemans J, Vandenneucker H. Septic arthritis after arthroscopic anterior cruciate ligament reconstruction: a retrospective analysis of incidence, management and outcome. Am J Sports Med 2007;35(7):1059-1063.

12. Schollin-Borg M, Michaëlsson K, Rahme H. Presentation, outcome, and cause of septic arthritis after anterior cruciate ligament reconstruction: a case control study. Arthroscopy 2003; 19(9):941-947.
13. Judd D, Bottoni C, Kim D, Burke M, Hooker S. Infections following arthroscopic anterior cruciate ligament reconstruction. Arthroscopy 2006;22(4):375-384.

14. Indelli PF, Dillingham M, Fanton G, Schurman DJ. Septic arthritis in postoperative anterior cruciate ligament reconstruction. Clin Orthop Relat Res 2002;398:182-188.

15. Abdel-Aziz A, Radwan YA, Rizk A. Multiple arthroscopic debridement and graft retention in septic knee arthritis after ACL reconstruction: a prospective case-control study. Int Orthop 2014:38(1):73-82.

16. Benner RW, Shelbourne KD, Freeman $H$. Infections and patellar tendon ruptures after anterior cruciate ligament reconstruction: a comparison of ipsilateral and contralateral patellar tendon autografts. Am J Sports Med 2011;39(3):519-525.

17. Binnet MS, Başarir K. Risk and outcome of infection after different arthroscopic anterior cruciate ligament reconstruction techniques. Arthroscopy 2007;23(8):862-868.

18. Burks RT, Friederichs MG, Fink B, Luker MG, West HS, Greis $\mathrm{PE}$. Treatment of postoperative anterior cruciate ligament infections with graft removal and early reimplantation. Am J Sports Med 2003;31(3):414-418.

19. Fong SY, Tan JL. Septic arthritis after arthroscopic anterior cruciate ligament reconstruction. Ann Acad Med Singap 2004;33(2): 228-234.

20. Jameson SS, Dowen D, James P, Serrano-Pedraza I, Reed MR, Deehan D. Complications following anterior cruciate ligament reconstruction in the English NHS. Knee 2012;19(1):14-19.

21. Maletis GB, Inacio MC, Funahashi TT. Analysis of 16,192 anterior cruciate ligament reconstructions from a communitybased registry. Am J Sports Med 2013;41(9):2090-2098.

22. Sechriest VF II, Carney JR, Kuskowski MA, Haffner IL, Mullen MJ, Covey DC. Incidence of knee sepsis after ACL reconstruction at one institution: the impact of a clinical pathway. J Bone Joint Surg Am 2013;95(9):843-846.

23. Viola R, Marzano N, Vianello R. An unusual epidemic of Staphylococcus-negative infections involving anterior cruciate ligament reconstruction with salvage of the graft and function. Arthroscopy 2000;16(2):173-177.

24. Wang C, Ao Y, Wang J, Hu Y, Cui G, Yu J. Septic arthritis after arthroscopic anterior cruciate ligament reconstruction: a retrospective analysis of incidence, presentation, treatment, and cause. Arthroscopy 2009;25(3):243-249.

25. Williams RJ III, Laurencin CT, Warren RF, Speciale AC, Brause BD, O'Brien S. Septic arthritis after arthroscopic anterior cruciate ligament reconstruction: diagnosis and management. Am I Sports Med 1997;25(2):261-267.

26. Schub DL, Schmitz LM, Sakamoto FA, Winalski CS, Parker RD. Long-term outcomes of postoperative septic arthritis after anterior cruciate ligament reconstruction. Am J Sports Med 2012; 40(12):2764-2770.

27. Mouzopoulos G, Fotopoulos VC, Tzurbakis M. Septic knee arthritis following ACL reconstruction: a systematic review. Knee Surg Sports Traumatol Arthrosc 2009;17(9):1033-1042.

28. Scully WF, Fisher SG, Parada SA, Arrington EA. Septic arthritis following anterior cruciate ligament reconstruction: a comprehensive review of the literature. J Surg Orthop Adv 2013;22(2): 127-133.

29. Sands K, Vineyard G, Platt R. Surgical site infections occurring after hospital discharge. J Infect Dis 1996;173(4):963-970.

30. Centers for Disease Control and Prevention. National Health- 
care Safety Network (NHSN) procedure-associated (PA) module: surgical site infection (SSI) event. http://www.cdc.gov/nhsn /PDFs/pscManual/9pscSSIcurrent.pdf. Accessed November 14, 2013.

31. Li X, King C, deGara C, White J, Winget M. Validation of colorectal cancer surgery data from administrative data sources. BMC Med Res Methodol 2012;12:97.
32. Centers for Medicare and Medicaid Services. Global surgery fact sheet. http://www.cms.gov/Outreach-and-Education/Medicare -Learning-Network-MLN/MLNProducts/downloads /GloballSurgery-ICN907166.pdf. Published August 2013. Accessed November 14, 2013. 